\title{
The case for extended lifespan in cooperatively breeding mammals: a re-appraisal
}

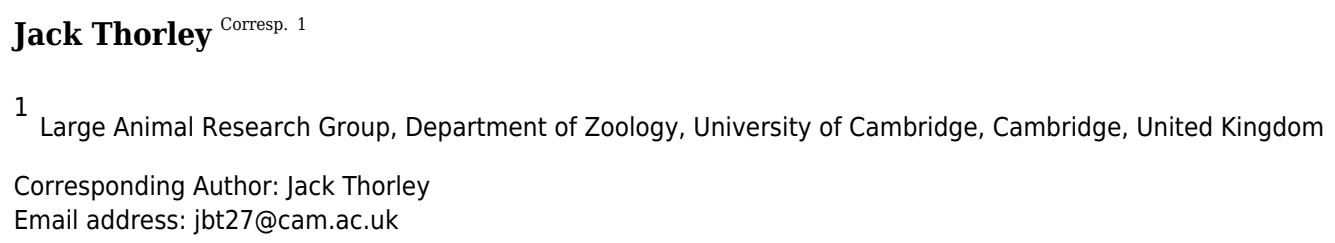

Recent comparative studies have suggested that cooperative breeding is associated with increases in maximum lifespan among mammals, replicating a pattern also seen in birds and insects. In this study, we re-examine the case for increased lifespan in mammalian cooperative breeders by analysing a large dataset of maximum longevity records. We did not find any consistent, strong evidence that cooperative breeders have longer lifespans than other mammals after having controlled for variation in body mass, mode of life and data quality. The only possible exception to this general trend is found in the African molerats (the Bathyergid family), where all members are relatively long-lived, but where the social, cooperatively breeding species appear to be much longer-lived than the solitary species. However, solitary mole-rat species have rarely been kept in captivity or followed longitudinally in the wild and so it seems likely that their maximum lifespan has been underestimated when compared to the highly researched social species. Although few subterranean mammals have received much attention in a captive or wild setting, current data instead supports a causal role of subterranean living on lifespan extension in mammals. 


\title{
The case for extended lifespan in cooperatively breeding mammals: a re-appraisal
}

\author{
Jack Thorley ${ }^{1}$ \\ ${ }^{1}$ Large Animal Research Group, Department of Zoology, University of Cambridge, United \\ Kingdom. \\ Corresponding Author \\ Jack Thorley \\ Large Animal Research Group, Department of Zoology, University of Cambridge, United \\ Kingdom. \\ Email address: jbt27@,cam.ac.uk
}

\begin{abstract}
Recent comparative studies have suggested that cooperative breeding is associated with increases in maximum lifespan among mammals, replicating a pattern also seen in birds and insects. In this study, we re-examine the case for increased lifespan in mammalian cooperative breeders by analysing a large dataset of maximum longevity records. We did not find any consistent, strong evidence that cooperative breeders have longer lifespans than other mammals after having controlled for variation in body mass, mode of life and data quality. The only possible exception to this general trend is found in the African mole-rats (the Bathyergid family), where all members are relatively long-lived, but where the social, cooperatively breeding species appear to be much longer-lived than the solitary species. However, solitary mole-rat species have rarely been kept in captivity or followed longitudinally in the wild and so it seems likely that their maximum lifespan has been underestimated when compared to the highly researched social species. Although few subterranean mammals have received much attention in a captive or wild setting, current data instead supports a causal role of subterranean living on lifespan extension in mammals.
\end{abstract}

Keywords: ageing; batherygids; comparative approach; cooperative breeding; eusociality; fossoriality; lifespan; sociality 


\section{Introduction}

Comparative studies of birds and insects have shown that species with cooperative and eusocial breeding systems have extended lifespans (Arnold \& Owens, 1998; Downing et al., 2015; Keller \& Genoud, 1997; Keller, 1998; see also Beauchamp, 2014). A common explanation for the association between these breeding systems and increased lifespan is that group-living reduces the extrinsic mortality of breeding individuals, which selects for greater longevity (Lucas and Keller, 2019). This is thought to be the case in eusocial insect societies where the 'queens' of many ant, bee and termite species live in a sheltered nest that is defended against predators by a large workforce (Carey, 2001; Hölldobler \& Wilson, 1990). An alternative possibility is that high annual survival increases local breeding competition, leading to delayed dispersal, family-living, and helping (Brown, 1987; Griesser et al., 2017), such that having a relatively long lifespan makes cooperative breeding more likely to evolve, and phylogenetic reconstructions of cooperative breeding supports this argument for birds (Downing et al., 2015).

Observations from certain mammals are also suggestive of a relationship between cooperative breeding and lifespan. The social mole-rats (family Bathyergidae), for example, include some of the longest-lived mammals for their size, with the naked mole-rat Heterocephalus glaber, a $40 \mathrm{~g}$ species from the Horn of Africa providing the most extreme case. In this species, breeding females can live for more than three decades in captivity and show no apparent agerelated changes in physiology or mortality rate (Buffenstein, 2005; Ruby et al., 2018; Ruby et al., 2019; though Dammann et al., 2019 have questioned whether the absence of Gompertzian mortality detected by Ruby et al. 2018 could have arisen from the structure of their data set, rather 
57 than representing a true biological effect), prompting much interest in naked mole-rats as a model

58

59

60

61

62

63

64 organism for gerontological research. However, as well as being long-lived, mole-rats are completely subterranean and are thought to seldom be exposed to predators in their burrow systems (Dammann \& Burda, 2007; Sándor, 2017), raising the question of whether it is their subterranean lifestyle, their cooperative breeding, or both that contribute to their longevity.

Two previous comparative analyses sought to separate the role of sociality and subterranean living on maximum lifespan in mammals, the first by Williams \& Shattuck (2015), and the second by Healy (2015). These studies followed on from an earlier comprehensive analysis of lifespan variation carried out by Healy et al. (2014) which tested whether species that possess traits thought to reduce extrinsic mortality risk are longer lived. This initial analysis identified fossoriality (defined as 'species living in permanent burrows') as one of several traits associated with increased maximum lifespan, with fossorial species living longer than semi-fossorial and nonfossorial species after correcting for phylogeny and body size. Yet despite being performed across a large number of non-flying animals, only five species in this first analysis were categorised as fossorial, including the long-lived naked mole-rat. In response to this one specific result, Williams and Shattuck (2015) noted that although it is logically consistent for underground living to be associated with increased lifespan, the sociality of the mole-rats could also account for their prolonged lifespan and obscure the interpretation of any fossoriality effect if both traits were not assessed concurrently.

To test their hypothesis, Williams and Shattuck (2015) collated a new dataset of maximum lifespan records from ground-living mammals and defined a larger number of species on the basis of their fossoriality (either terrestrial or fossorial) and their sociality ('eusocial' or not). While Williams and Shattuck demarcated sociality on the basis of 'eusocial tendencies', we would rather 
80 refer to such species as cooperative breeders to avoid the semantic difficulties that arise when

81 applying a definition of eusociality to a wide range of taxa (Boomsma \& Gawne 2017; Burda et

82 al., 2000). Cooperative breeding can be more accurately defined as those species where a

83 proportion of females do not breed regularly and have been shown to perform alloparental care in

84 the form of direct or indirect provisioning of offspring (as per Solomon \& French, 1997; Lukas \&

85 Clutton-Brock, 2012a). Analyses of phylogenetically independent contrasts on the new dataset

86 suggested that both fossoriality and sociality (cooperative breeding) were associated with increases

87 in maximum lifespan, but by the authors' own admission, more nuanced phylogenetic approaches

88 could provide a more robust assessment of these patterns. Following this recommendation, Healy

89 (2015) re-analysed the dataset of Williams and Shattuck (2015) using a Bayesian approach that

90 accounts for phylogenetic uncertainty by combining information from models fitted across

91 multiple mammalian trees, and doing so, found that that sociality, and not fossoriality, drove

92 increases in maximum lifespan in mammals.

93 In this study, we re-visit the question of whether sociality, fossoriality, or both, are related

94 to increases in maximum lifespan. The intention is not to detract from the results of the previous

95 studies, but to place these results in context, as the ongoing reference to a link between sociality

96 and increased lifespan in mammals (e.g. Downing et al. 2015; Lucas \& Keller, 2019) could give

97 the perception of a robust and resolved trend in spite of an equivocal evidence base. Indeed, not

98 all published studies have demonstrated a positive effect of cooperative breeding on maximum

99 lifespan. Lukas and Clutton-Brock (2012a) compared cooperatively breeding mammals to socially

100 monogamous mammals and found no clear difference in maximum lifespan between species with

101 these two mating systems. As cooperative breeding is sparsely represented across the mammalian

102 clade (less than 1\% of mammals, Lukas \& Clutton-Brock, 2012b), slight differences in data 
103 availability or analytical method, or contrasts in definitions could contribute to inconsistencies in

104 results across studies, and it is therefore worthwhile reflecting on how and why different studies

105 might come to reach different conclusions. To this end, we build on previous work by using a more

106 stringent definition of sociality along with a definition of fossoriality more aligned with the

107 original definition in Healy et al. (2014) to test the role of cooperative breeding and fossoriality in

108 driving increases in maximum lifespan. Although the use of maximum lifespan records as a proxy

109 for lifespan is not without criticism (e.g. Baylis et al., 2014), the absence of detailed life table

110 information for many species usually precludes the use of alternative ageing metrics in a 111 comparative setting. The use of lifespan information from captive animals could also be criticised.

112 Clearly, captivity provides a safe environment where individuals are buffered from environmental

113 stochasticity, predation, and resource competition, and veterinary care largely eliminates disease

114 and morbidity (Tidière et al., 2016). Studies of senescence in captive populations therefore make

115 the assumption that demographic features of senescence in captivity, including maximum lifespan,

116 are a product of (or are proportional to) selection on mortality in the wild and therefore provide

117 useful information on the intrinsic deterioration of a species (Carey 2003; De Magalhães et al., 118 2007).

Our approach expands upon the two previous treatments of this topic in several important respects. The first major difference is in the categorisation of species according to their use of the underground environment. Here, we separate species according to whether they are subterranean

122 feeders or not. Subterranean feeders spend almost their entire lives underground and are therefore 123 likely to experience the low levels of predation and extrinsic mortality that should select for longer

124 lifespans (Hartman, 1995; Novikov \& Burda, 2013). In contrast, Williams and Shattuck (2015) 125 chose a more relaxed definition, subsequently followed by Healy (2015), whereby all mammals 
126 that make extensive use of the underground environment were classed as fossorial. This definition

127 forces one to group subterranean feeders (such as the moles, mole-rats, pocket gophers, or coruro

128 Spalacopus cyanus) with species that use the underground environment extensively for resting,

129 denning, and sometimes food storage, but which otherwise spend a large part of their daily activity

130 period foraging above-ground (for example armadillos, aardvark Orcyteropus afer, or Eurasian

131 badger Meles meles). It is not clear that mammals falling into this latter category should experience

132 reduced predation rates to the same extent as subterranean feeders (see Healy et al., 2014 for

133 similar interpretation), and grouping the two classes could therefore prevent the detection of an

134 effect of subterranean living on lifespan. Secondly, although previous studies tried to overcome

135 possible issues of sample size by using sensitivity analyses- either by removing outliers with small

136 sample sizes (Williams \& Shattuck 2015), or by comparing the results of analyses where species

137 with low sample size were included or excluded (Healy et al. 2014)- they did not explicitly include

138 sample size in their modelling framework. It is known that a large number of lifespan records are

139 needed before a maximum lifespan estimate begins to asymptote (Moorad et al., 2012), and since

140 cooperative breeders are more frequently studied and kept in captivity, then all else being equal,

141 the maximum lifespans of cooperative breeders are anticipated to be greater than non-

142 cooperatively breeding species for purely numerical reasons (see methods); so sample size needs

143 to be controlled for and estimated alongside the effect of cooperative breeding (e.g. Kamilar et al.,

144 2010; Minias \& Podlaszczuk, 2017). Thirdly, by focussing on ground-dwelling (i.e. non-arboreal)

145 mammals, previous studies have not considered lifespan data from the callitrichid primates (the

146 marmosets and tamarins), which represent a sizeable fraction of the total pool of cooperatively

147 breeding species. Here, we include information from callitrichid primates. 
149 for sociality, subterranean living, mode of life, data quality, and body mass. To account for

150 phylogenetic uncertainty the analysis was performed in a Bayesian framework that combined

151 information from multiple mammalian trees. We then repeated the analysis on different subsets of

152 the global dataset to investigate the possible influence of outlier species and data structure on 153 estimated effect sizes.

\section{Material and Methods}

\section{Global dataset} species (Figure 1; De Magalhães \& Costa, 2009). This dataset provides estimates of the sample size for each longevity record, reflecting orders of magnitude in the number of specimens that contributed to the record. Species with fewer than 10 specimens contributing to their record ('tiny') were excluded. The only exception to this data restriction was the Cape mole-rat Georychus capensis, which was retained because of the general lack of longevity data for African mole-rats, family Bathyergidae; for model fitting purposes this species was reclassified as having 'small' sample size. This left 323 species with a 'small' sample size (10-100 specimens), 272 species with a medium sample size (100-1000), and 123 species with a 'large sample size' (over 1000 specimens). Of these 719 longevity records, 712 originated from captive populations (99.0\%), 3 originated from wild populations, and 3 were of unknown origin. Sampling was not evenly distributed across cooperative and non-cooperative species: for cooperative species, large, medium and small lifespan sample sizes reflected $36.7 \%, 40.0 \%$ and $23.3 \%$ of species, as compared to $16.2 \%, 37.8 \%$ and $46.0 \%$ for non-cooperative species $\left(\chi^{2}{ }_{2}=10.38, \mathrm{p}=0.006\right)$. All else being equal, 
171 longevity records for cooperative breeders are therefore likely to be higher simply due to sampling

172 effort. Species that have been documented to breed cooperatively were defined as in the

173 introduction $(n=30$ cooperative breeders). For each species further information was added on

174 adult body mass, subterranean living and habitat. Subterranean living demarcated species as

175 subterranean or non-subterranean feeders using information from several sources (Begall et al.,

176 2007; Mittermeier et al., 2018; n = 13 subterranean species). For 'habitat', species were defined

177 as arboreal $(n=150)$, semi-arboreal $(n=70)$ or ground-dwelling $(n=499)$, using information from

178 Walker's Mammals of the World (Nowak, 1999) and the Handbook of the Mammals of the World

179 (Mittermeier et al., 2018).

180 Global analysis

To investigate the influence of the chosen predictors on maximum lifespan, a global model was fitted which took the form:

$\ln ($ maximum lifespan $) \sim \ln ($ adult body mass $)+$ cooperative breeding +

subterranean + lifestyle + lifespan sample size

Adult body mass was z-score transformed before model fitting. We implemented the model

as a Bayesian phylogenetic mixed model (PLMM) using the MCMCglmm package (Hadfield, 2010) in R v3.6.2. PLMMs account for nonindependence between species by incorporating the phylogenetic tree as a random effect (Hadfield \& Nakagawa, 2010). To account for phylogenetic uncertainty, we fitted the model on 100 randomly chosen mammalian trees from Kuhn et al. (2011) and extracted the mean density of the combined posterior distribution using the mulTree Package

191 (Guillerme \& Healy, 2015). For each tree we ran two chains of 105000 iterations with a burn-in 192 of 5000 and a thinning interval of 100 (generating a total 2000 posterior samples for each parameter 
193 per tree). An inverse Wishart prior was chosen for the variance components $(\mathrm{V}=1, \mathrm{nu}=0.02)$.

194 All fitted models converged and the Gelman-Rubin statistic was always $<1.1$ for all parameters

195 (Gelman \& Rubin, 1992). The fixed effects were considered biologically significant when the 95\%

196 credible interval did not span zero. The marginal and condition $R^{2}$ for the model were calculated

197 according to Nakagawa \& Schielzeth (2013).

To explore how model estimates were affected by the composition of the global dataset, we repeated the modelling approach on several subsets of the main dataset. We first repeated the analysis by excluding the naked mole-rat, or the Cape mole-rat. The naked mole-rat is a known outlier for mammalian longevity (Buffenstein, 2005), so a stronger case for a general effect of cooperative breeding on lifespan could be made if a cooperative breeding trend were present in the absence of this species. We also repeated the analysis without the Cape mole-rat to confirm that our inclusion of this species - with very low sample size informing the longevity record - did not unduly effect model estimates. We then re-ran the analyses to only include ground-dwelling species (i.e. excluded arboreal and semi-arboreal species to yield a dataset with $n=499$ (with $n=$ 16 cooperative breeders)), and also conducted a smaller analysis that only included five mammalian families that contain both cooperative and non-cooperatively breeding species (the mongooses; generating a dataset of 100 species, of which $n=27$ were cooperative breeders).

211 Because of the reduced dataset size for this latter analysis we only included fixed effects of 212 cooperative breeding status, body mass and sample size. 
215 Across all the phylogenetic models fitted to the various datasets cooperative breeding had a 216 positive effect on maximum lifespan (Table 1), but in all cases the effect size was small - reflecting 217 at most a 3.1\% increase in lifespan - and non-significant (Figure 1, Table 1, Table S1). In contrast, 218 subterranean feeding consistently explained significant increases in maximum lifespan across the 219 candidate models (Figure 1, Table 1, Table S1), and the influence of this term was maintained even 220 after the removal of the known outlier species, the naked mole-rat. The effect of subterranean 221 feeding ranged from $9.9 \%$ when the naked mole-rat was absent, to $12.8 \%$ when present. Beyond 222 the effects of sociality and subterranean living, the models recovered a clear influence of sample 223 size, such that species with more records contributing to their maximum lifespan had greater 224 reported lifespans, all else being equal. The models also recovered the expected positive 225 association between body size and maximum lifespan, (with the exception of the cooperative 226 families dataset, Table 1, Table S1) and found that arboreal species were longer-lived than ground227 dwelling species, with semi-arboreal species somewhere intermediate between these other two 228 modes of life.

\section{Discussion}

The results presented in this study failed to find clear evidence for an association between cooperative breeding and increases in maximum lifespan across terrestrial mammals. Though the sample set of cooperatively breeding mammals is necessarily small, the effect size of any cooperative breeding effect on maximum lifespan is modest (at most 3.1\%), and as such, it seems and lifespan remains obscure, particularly when viewed in light of ageing patterns in social insects 
237 (Keller \& Genoud, 1997), or indeed birds (Arnold \& Owens, 1998). In contrast, subterranean

238 feeding was related to marked increases in maximum lifespan $(12.8 \%)$, and this effect remained

239 robust to the exclusion of the exceptional naked mole-rat. That subterranean species are longer-

240 lived is in line with evolutionary theories of senescence from which one can predict that lifestyles

241 that reduce the level of extrinsic mortality - as is expected for species permanently inhabiting a

242 subterranean niche- lead to the evolution of longer lifespans, and specific physiological adaptations

243 in several long-lived subterranean species supports this view (Kim et al., 2011; Fang et al., 2014).

244 Even so, there remains little quantitative information on the demography of subterranean taxa and

245 this should be borne in mind when extrapolating our result more generally.

A consideration of the social organisation of cooperative societies can make sense of the reported null effect of cooperative breeding on lifespan. As helpers buffer the costs of reproduction in females (e.g. Solomon \& French, 1997, Russell et al., 2003; Creel, Mills \& McNutt, 2004), it could be argued that helpers might increase maximum lifespan by lightening the costs of reproduction for breeders, and recent work in Seychelles warblers Acrocephalus sechellensis has shown that the presence of a subordinate helper increases late-life survival in breeding females (Hammers et al., 2019). However, in cooperatively breeding mammals, the presence of helpers has also led to evolution of unusually high reproductive rates, and in some cases, atypically large litter sizes (Barrette et al., 2012; Clutton-Brock et al., 2006; Clutton-Brock, 2016). In addition, the extreme reproductive skew apparent in cooperative societies often engenders intense competition over mating opportunities which manifests in reproductive suppression, high levels of aggression, and the incidence of infanticide (Clutton-Brock, 2016). The presence of intense reproductive competition and high rates of reproduction are typically associated with high levels of energy expenditure and would classically be expected to accelerate rates of ageing and reduce lifespan, 
260 not prolong it (Stearns, 1989). Indeed, in wild Kalahari meerkats, females that experienced greater

261 competition in early life displayed faster rates of reproductive senescence in later life (Sharp \&

262 Clutton-Brock, 2011). Previous work has also failed to find a link between group size and lifespan

263 in mammals (Kamilar et al., 2010), so it is also not clear whether the large group sizes of

264 cooperative breeders should necessarily contribute to their pace of life either.

265 The only family where cooperatively breeding species appear to live longer compared to 266 non-cooperative breeders is in the bathyergid mole-rats. Within this family the social taxa such as

267 the naked mole-rat (31-year maximum lifespan) and the Damaraland mole-rat Fukomys 268 damarensis (15.5 years), live markedly longer than the non-social, solitary members of the family

269 such as the Silvery mole-rat Heliophobius argentocinereus (7.5 years), or the Cape mole-rat

270 Georychus capensis (11.2 years). However, this comparison must be treated with caution, for

271 unlike the social species, solitary mole-rats have attracted much less interest from researchers and

272 are notoriously difficult to maintain in captivity, which will lead to large underestimates of the

273 longevity of solitary species. There are nonetheless a number of illustrative cases outside of the

274 African mole-rats that would tend to support a connection between subterranean living and lifespan

275 extension. The subterranean and solitary plains pocket gopher (Geomys bursarius) and the Middle

276 East blind mole rat (Nannospalax ehrenbergi) provide two examples, having been known to live

277 for 12 years and 15 years, respectively (Weigl, 2005).

278 While our results are more suggestive of a role of subterranean living on lifespan, few 279 subterranean mammals have been kept in captivity or been the focus of long-term individual-based 280 studies. Moreover, a recent comparative analysis of reptiles failed to find a significant effect of 281 fossoriality on lifespan (Stark et al., 2018), which the authors reasoned could be because any 282 reductions in predation risk brought about through fossoriality are offset by high metabolic costs 
283 of burrowing. Taken together it therefore seems premature to place judgement on the role of

284 subterranean living on ageing patterns in mammals until higher resolution data is collated from a

285 larger number of species which permanently inhabit a subterranean niche, both in the wild and in 286 captivity. cooperatively breeding mammals live longer than their non-cooperative counterparts. Whether this is true for other, more robust metrics of ageing will be an important test of the generality of this finding.

\section{Acknowledgements}

JT would like to thank Prof. Tim Clutton-Brock, Louis Bliard and three reviewers for their comments on the manuscript.

\section{In-Text Tables}

298

299

300

301

302

303

304
Table 1. Phylogenetic analyses of maximum lifespan across terrestrial mammals. Models were fitted using a phylogenetic linear mixed effects modelling framework, to a full dataset, a dataset excluding the naked mole-rat, and a dataset fitted to five cooperatively breeding families containing both cooperatively and non-cooperatively breeding species (Bathyergidae, Callitrichidae, Canidae, Cricetidae, Herpestidae). Estimates refers to the mean of the posterior distribution from models fitted to 100 different mammalian trees. Terms where the $95 \%$ credible intervals did not overlap zero were deemed biologically significant (highlighted in bold). The reference category refers to non-cooperatively breeding, ground-dwelling species. 
307 Figure Legends

308

309

310

311

312

313

314

315

316

317

318

319

320

321

322

323

324

325

326

327

328

329

330

331

332

333

Figure 1. The relationship between $\log _{\mathrm{e}}$ adult body mass and $\log _{\mathrm{e}}$ maximum lifespan for 719

terrestrial mammals. A- Species are coloured according to Order. Because of the strong phylogenetic contribution to maximum lifespan, the change in lifespan with increasing mass is predicted relative to the species with the median random effect estimate in each Order (as extracted from the MCMCglmm model fitted to the full dataset). Cooperative breeders and subterranean feeders have been given larger, highlighted points (cooperatively breeding only- bold circles; subterranean only- bold triangles; cooperatively breeding and subterranean- bold squares). B- The predicted effect of cooperative breeding (dotted line) and/or subterranean feeding (thick solid line) is only illustrated in Orders containing species that display either trait. The animal icons were taken from PhyloPic: http://phylopic.org. Predictions were generated at the medium sample size for a ground-dwelling mammal.

\section{References}

Arnold, K.E., \& Owens, I.P.F. 1998. Cooperative breeding in birds: a comparative test of the life history hypothesis. Proc. R. Soc. B. 265(1398): 739-745. doi:10.1098/rspb.1998.0355

Baylis, S.M., de Lisle, M., \& Hauber, M.E. 2014. Inferring maximum lifespan from maximum recorded longevity in the wild carries substantial risk of estimation bias. Ecography 37:770-778. doi:10.1111/ecog.00507.

Barrette, M.F., Monfort, S.L., Festa-Bianchet, M., Clutton-Brock, T.H., \& Russell, A.F. 2012. Reproductive rate, not dominance status, affects fecal glucocorticoid levels in breeding female meerkats. Horm. Behav. 61(4): 463-471. doi:10.1016/j.yhbeh.2011.12.005

Beauchamp, G. 2014. Do avian cooperative breeders live longer? Proc. R. Soc. B. 281(1787): 6-10. doi:10.1098/rspb.2014.0844

Begall, S., Burda, H., \& Schliech, C.E. 2007. Subterranean rodents: News from underground. Berlin Heidelberg: Springer Verlag.

Boomsma, J.J., \& Gawne, R. 2018. Superorganismality and caste differentiation as points of no return: how the major evolutionary transitions were lost in translation. Biol. Rev. 93:27-46. 
334 Brown, J.L. 1987. Helping and communal breeding in birds. Princeton University Press, Princeton.

335

336

337

338

339

340

341

342

343

344

345

346

347

348

349

350

351

352

353

354

355

356

357

358

359

360

361

362

363

364

365

366

367

Buffenstein, R. 2005. The naked mole-rat: a new long-living model for human aging research. J. Gerontol. Series A 60(11): 1369-77.

Burda, H., Honeycutt, R.L., Begall, S.B., Locker-Grütjen, O., \& Scharff. A. 2000. Are naked and common mole-rats eusocial and if so, why? Behav. Ecol. Sociobiol. 47(5): 293-303.

Carey, J.R. 2001. Demographic mechanisms for the evolution of long life in social insects. Exp. Gerontol. 36: 713-22. doi:10.1016/S0531-5565(00)00237-0.

Carey, J.R. 2003. Longevity. The biology and demography of lifespan. Princeton, New Jersey: Princeton University Press.

Clutton-Brock, T. 2016. Cooperative Breeding. In Mammalian Societies (T. Clutton-Brock, eds), pp. 557604. Chichester, UK: John Wiley \& Sons Ltd.

Clutton-Brock, T.H., Hodge, S.J., Spong, G., Russell, A.F., Jordan, N.R., Bennett, N.C., Sharpe, L.L., \& Manser, M.B. 2006. Intrasexual competition and sexual selection in cooperative mammals. Nature 444(7122): 1065-8. doi:10.1038/nature05386.

Creel, S., Mills, M.G.L., McNutt, J.W. 2004. Demography and population dynamics of African wild dogs in three critical populations. In: Macdonald, D.W., Sillero-Zubiri, C., editors. Biology and conservation of wild canids. Oxford, UK: Oxford University Press, p. 337-350.

Dammann, P., \& Burda, H. 2007. Senescence patterns in African mole-rats (Bathyergidae, Rodentia). In: Subterranean rodents: News from underground (Begall, S., Burda, H., \& Schliech, C.E , eds), pp. 251-263. Berlin Heidelberg: Springer Verlag.

Dammann, P., Scherag, A., Zak, N., Szafranski, K., Holtze, S., Begall, S., Burda, H., Kestler, H., Hildebrandt, T., \& Platzer, M. 2019. Comment on 'Naked mole-rat mortality defy Gompertzian laws by not increasing with age'. eLife 8:e45415.

Downing, P.A., Cornwallis, C.K., \& Griffin, A.S. 2015. Sex, long life and the evolutionary transition to cooperative breeding in birds. Proc. R. Soc. B. 282: 1-7. doi: 10.1098/rspb.2015.1663

Fang, X., Seim, I., Huang, Z., Gerashchenko, M.V, Xiong, Z., Turanov, A.A., Zhu, Y., Lobanov, A.V., Fan, D., Yim, S.H., Yao, X., Ma, S., Yang, L., Lee, S., Kim, E.B., Bronson, R.T., Šumbera, R., Buffenstein, R., Zhou, X., Krogh, A., Park, T.J., Zhang, G., Wang, J., \& Gladyshev, V.N. 2014. Adaptations to a subterranean environment and longevity revealed by the analysis of mole rat genomes. Cell rep. 8(5): 1354-64. doi:10.1016/j.celrep.2014.07.030

Gelman, A. \& Rubin, D.B. 1992. Inference from iterative simulation using multiple sequences. Stat Sci 7(4): 457-472.

Griesser, M., Drobniak, S.M., Nakagawa, S., \& Botero, C.A. 2017. Family living sets the stage for cooperative breeding and ecological resilience in birds. PLoS Biol. 15(6): 1-17.

Peer) reviewing PDF | (2019:09:41283:2:0:NEW 21 Apr 2020) 
doi:10.1371/journal.pbio.2000483

Guillerme, T., \& Healy, K. 2014. mulTree: a package for running MCMCglmm analysis on multiple trees. See https://doi.org/10.5281/zenodo.12902.

Hadfield, J. 2010. MCMC methods for Multi-Response Generalized Linear Mixed Models: The MCMCglmm R Package. J. Stat. Softw. 33(2): 1-22.

Hadfield, J.D., \& Nakagawa, S. 2010. General quantitative genetic methods for comparative biology: Phylogenies, taxonomies and multi-trait models for continuous and categorical characters. J. Evol. Biol. 23(3): 494-508. doi:10.1111/j.1420-9101.2009.01915.x

Hammers, M., Kingma, S.A., Spurgin, L.G., Bebbington, K., Dugdale, H.L., Burke, T., Komdeur, J., \& Richardson, D.S. 2019. Breeders that receive help age more slowly in a cooperatively breeding bird. Nat. Comms. doi: 10.1038/s41467-019-09229-3.

Hartman, G.D. 1995. Age determination, age structure, and longevity in the mole, Scalopus aquaticus (Mammalia: Insectivora). J. Zool. 237: 107-122.

Healy, K. 2015. Eusociality but not fossoriality drives longevity in small mammals. Proc. R. Soc. B. 282, 20142917. doi:10.5281/zenodo.12902

Healy, K., Guillerme, T., Finlay, S., Kane, A., Kelly, S. B., McClean, D., Kelly, D.J., Donohue, I., Jackson, A.L., \& Cooper, N. 2014. Ecology and mode-of-life explain lifespan variation in birds and mammals. Proc. R. Soc. B. 281: 20140298.

Hölldobler, B., \& Wilson, E.O. 1990. The Ants. Berlin: Springer.

Kamilar, J.M., Bribiescas, R.G., \& Bradley, B.J. 2010. Is group size related to longevity in mammals? Biol. Lett. 6(6): 736-739. doi:10.1098/rsbl.2010.0348

Keller, L., \& Genoud, M. 1997. Extraordinary lifespans in ants: a test of evolutionary theories of ageing. Nature 389: 958-960. doi:10.1038/40130

Keller, L. 1998. Queen lifespan and colony characteristics in ants and termites. Insectes sociaux 45(3): 235246.

Kim, E.B., Fang, X., Fushan, A.A., Huang, Z., Lobanov, A.V, Han, L., Marino, S.M., Sun, X., Turanov, A.A., Yang, P., Yim, S.H., Zhao, X., Kasaikina, M.V., Stoletzki, N., Peng, C., Polak, P., Xiong, Z., Kiezun, A., Zhu, Y., Chen, Y., Kryukov, G.V., Zhang, Q., Peshkin, L., Yang, L., Bronson, R.T., Buffenstein, R., Wang, B., Han, C., Li, Q., Chen, L., Zhao, W., Sunyaev, S.R., Park, T.J., Zhang, G., Wang, J., \& Gladyshev, V.N. 2011. Genome sequencing reveals insights into physiology and longevity of the naked mole rat. Nature 479(7372): 223-7. doi:10.1038/nature10533

Kuhn, T.S., Mooers, A.O., \& Thomas, G.H. 2011. A simple polytomy resolver for dates phylogenies. Methods Ecol. Evol. 2, 427-436. doi: 10.1111/j.2041-210X.2011.00103.x

Lucas, E.R. \& Keller, L. 2019. The co-evolution of longevity and social life. Funct. Ecol. doi: 
402

403

404

405

406

407

408

409

410

411

412

413

414

415

416

417

418

419

420

421

422

423

424

425

426

427

428

429

430

431

432

433

434

435

$10.1111 / 1365-2435.13445$.

Lukas, D., \& Clutton-Brock, T. 2012a. Life histories and the evolution of cooperative breeding in mammals. Proc. R. Soc. B. 279(1744): 4065-70. doi:10.1098/rspb.2012.1433

Lukas, D., \& Clutton-Brock, T. 2012b. Cooperative breeding and monogamy in mammalian societies. Proc. R. Soc. B. 279(1736): 2151-6. doi:10.1098/rspb.2011.2468

Lukas, D., \& Clutton-Brock, T. 2017. Climate and the distribution of cooperative breeding in mammals. Royal Soc. Open Sci. 4(1): 160897. doi:10.1098/rsos.160897.

de Magalhães, J. P., Costa, J., \& Church, G.M. 2007. An analysis of the relationship between metabolism, developmental schedules, and longevity using phylogenetic independent contrasts. cords and their relation to other life-history traits. J. Gerontol. A Biol. Sci. Med. Sci. 62(2): 149-160. doi:10.1093/gerona/62.2.149.

de Magalhães, J. P., \& Costa, J. 2009. A database of vertebrate longevity records and their relation to other life-history traits. J. Evol. Biol. 22(8): 1770-1774. doi:10.1111/j.1420-9101.2009.01783.x

Minias, P., \& Podlaszczuk, P. 2017. Longevity is associated with relative brain size in birds. Ecol. Evol. 7(10): 3558-3566. doi:10.1002/ece3.2961.

Mittermeier, R.A., Rylands, A.B., \& Wilson, D.E. 2018. Handbook of the Mammals of the World. Barcelona, Spain: Lynx Edicions.

Moorad, J.A., Promislow, D.E.L., Flesness, N., \& Miller, R.A. 2012. A comparative assessment of univariate longevity measures using zoological animal records. Aging Cell 11(6): 940-948. doi:10.1111/j.1474-9726.2012.00861.x

Nakagawa, S., Schielzeth, H. 2013. A general and simple method for obtaining R2 from generalized linear mixed-effects models. Methods Ecol. Evol. 4: 133-142. doi: 10.111/j.2041-210x.2012.00261.x

Nowak, R.M. 1999. Walker's mammals of the world. John Hopkins University Press, Baltimore.

Novikov, E.A., \& Burda, H. 2013. Ecological and evolutionary preconditions of extended longevity in subterranean rodents. Biology Bulletin Reviews 3(4): 325-333.

Ruby, J.G., Smith, M., \& Buffenstein, R. 2018. Naked mole-rat mortality rats defy gompertzian laws by not increasing with age. eLife 7: 1-18. doi:10.7554/eLife.31157.

Ruby, J.G., Smith, M., \& Buffenstein, R. 2019. Response to comment on "Naked mole-rat mortality rates defy Gompertzian laws by not increasing with age". eLife 8, e47047. doi:10.7554/eLife.47047.

Russell, A.F., Brotherton, P.N.M., McIlrath, G.M., Sharpe, L.L., \& Clutton-Brock, T.H. 2003. Breeding success in cooperative meerkats: effects of helper number and maternal state. Behav. Ecol. 14: 486492.

Sándor, A.D. 2017. Underground life is still safest: comments on "Danger underground and in the open predation on blind mole rats (Rodentia, Spalacinae) revisited." Mammal Rev. 47(3): 230-235. 
436

437

438

439

440

441

442

443

444

445

446

447

448

449

450

doi:10.1111/mam.12089

Sharp, S.P., \& Clutton-Brock, T.H. 2011. Competition, breeding success and ageing rates in female meerkats. J. Evol. Biol. 24: 1756-1762.

Solomon, N.G., \& French, J.A. 1997. Cooperative Breeding in Mammals. Cambridge, UK: Cambridge University Press.

Stark, G., Tamar, K., Itescu, Y., Feldman, A., Meiri, S. 2018. Cold and isolated ectotherms: drivers of reptilian longevity. Biol. J. Linn. Soc. 125: 730-740.

Stearns, S. 1989. Trade-offs in life-history evolution. Funct. Ecol. 3(3), 259-268.

Tidière, M, Gaillard, J.-M., Berger, V., Müller, D.W.H., Lackey, L.B., Gimenez, O., Clauss, M, \& Lemaître, J.-F. 2016. Comparative analyses of longevity and senescence reveal variable survival benefits of living in zoos across mammals. Scientific Reports 6, 36361. doi: 10.1038/srep36361.

Weigl, R. 2005. Longevity of mammals in captivity: from the living collections of the world. A list of mammalian longevity in captivity. Stuttgart: Schweizerbart.

Williams, S.A., \& Shattuck, M.R. 2015. Ecology, longevity and naked mole-rats: confounding effects of sociality? Proc. R. Soc. B 282: 20141664. doi: 10.1098/rspb.2014 


\section{Table $\mathbf{1}$ (on next page)}

Phylogenetic analyses of maximum lifespan across terrestrial mammals

Phylogenetic analyses of maximum lifespan across terrestrial mammals. Models were fitted using a phylogenetic linear mixed effects modelling framework, to a full dataset, a dataset excluding the naked mole-rat, and a dataset fitted to five cooperatively breeding families containing both cooperatively and non-cooperatively breeding species (Bathyergidae, Callitrichidae, Canidae, Cricetidae, Herpestidae). Estimates refers to the mean of the posterior distribution from models fitted to 100 different mammalian trees. Terms where the 95\% credible intervals did not overlap zero were deemed biologically significant (highlighted in bold). The reference category refers to non-cooperatively breeding, ground-dwelling species. 
Table 1. Phylogenetic analyses of maximum lifespan across terrestrial mammals. Models were fitted using a phylogenetic linear mixed effects modelling framework, to a full dataset, a dataset excluding the naked mole-rat, and a dataset fitted to five cooperatively breeding families containing both cooperatively and non-cooperatively breeding species (Bathyergidae, Callitrichidae, Canidae, Cricetidae, Herpestidae). Estimates refers to the mean of the posterior distribution from models fitted to 100 different mammalian trees. Terms where the $95 \%$ credible intervals did not overlap zero were deemed biologically significant (highlighted in bold). The reference category refers to non-cooperatively breeding, ground-dwelling species.

\begin{tabular}{|c|c|c|c|}
\hline Model Term & $\begin{array}{c}\text { Global Dataset } \\
\text { Mean estimate }(95 \% \mathrm{CI})\end{array}$ & $\begin{array}{c}\text { Global dataset without } \\
\text { naked mole-rat } \\
\text { Mean estimate }(95 \% \mathrm{CI})\end{array}$ & $\begin{array}{c}\begin{array}{c}\text { Cooperatively breeding } \\
\text { families }\end{array} \\
\text { Mean estimate }(95 \% \mathrm{CI})\end{array}$ \\
\hline Intercept & $2.724(2.123-3.324)$ & $2.727(2.136-3.320)$ & $2.411(2.032-2.790)$ \\
\hline Adult body mass & $0.332(0.275-0.391)$ & $0.339(0.282-0.370)$ & $0.096(-0.029-0.222)$ \\
\hline Cooperative breeding status: cooperative & $0.065(-0.200-0.149)$ & $0.056(-0.029-0.141)$ & $0.061(-0.033-0.156)$ \\
\hline Fossoriality: subterranean & $0.348(0.093-0.603)$ & $0.269(0.012-0.526)$ & - \\
\hline Lifestyle: semi-arboreal & $0.083(-0.009-0.175)$ & $0.084(-0.007-0.175)$ & - \\
\hline Lifestyle: arboreal & $0.129(0.024-0.235)$ & $0.131(0.026-0.235)$ & - \\
\hline Sample size: medium & $0.127(0.089-0.166)$ & $0.126(0.088-0.165)$ & $0.118(0.027-0.204)$ \\
\hline Sample size: large & $0.207(0.154-0.259)$ & $0.202(0.149-0.254)$ & $0.287(0.158-0.414)$ \\
\hline $\begin{array}{l}\text { Residual variance } \\
\text { Phylogenetic variance }\end{array}$ & $\begin{array}{l}0.015(0.010-0.021) \\
0.388(0.314-0.464)\end{array}$ & $\begin{array}{l}0.015(0.010-0.021) \\
0.378(0.303-0.453)\end{array}$ & $\begin{array}{l}0.015(0.008-0.026) \\
0.165(0.096-0.274)\end{array}$ \\
\hline $\begin{array}{l}\text { Marginal R2 } \\
\text { Conditional R2 }\end{array}$ & $\begin{array}{l}0.258(0.190-0.336) \\
0.972(0.958-0.982)\end{array}$ & $\begin{array}{l}0.270(0.200-0.349) \\
0.971(0.957-0.982)\end{array}$ & $\begin{array}{l}0.138(0.044-0.290) \\
0.924(0.852-0.969)\end{array}$ \\
\hline Species number & 719 & 718 & 100 \\
\hline
\end{tabular}


Figure 1

The relationship between $\log _{e}$ adult body mass and $\log _{e}$ maximum lifespan for 719 terrestrial mammals.

The relationship between $\log _{\mathrm{e}}$ adult body mass and $\log _{\mathrm{e}}$ maximum lifespan for 719 terrestrial mammals. Species are coloured according to Order, and cooperative breeders have been given larger, highlighted points. An ordinary least-squares regression is fitted through each Order to indicate phylogenetic differences in the scaling relationship.
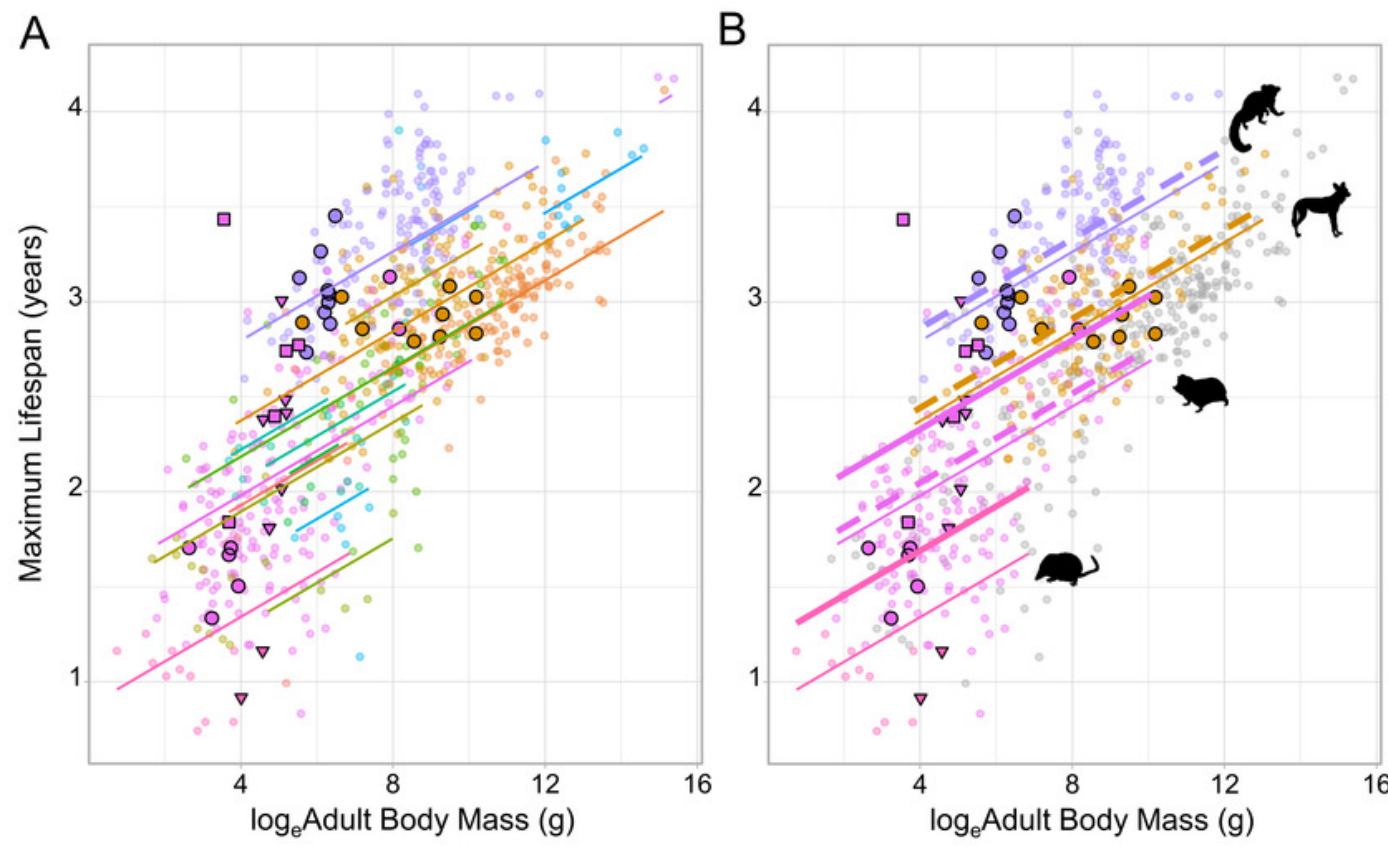

\begin{tabular}{ll}
\multicolumn{2}{c}{ Order } \\
OAfrosoricida & OMonotremata \\
OArtiodactyla & OPeramelemorphia \\
OCarnivora & OPerissodactyla \\
OCingulata & OPilosa \\
ODasyuromorphia & OPrimates \\
ODidelphimorphia & OProboscidea \\
ODiprotodontia & ORodentia \\
OErinaceomorpha & OScandentia \\
OHyracoidea & OSoricomorpha \\
OLagomorpha & OTubulidentata \\
OMacroscelidea &
\end{tabular}

